\title{
Unsteady stagnation-point boundary layer flows of power-law fluids over a porous flat plate
}

\author{
S. Dholey ${ }^{1}$ (D)
}

Received: 7 January 2020 / Accepted: 20 August 2020 / Published online: 31 August 2020

(c) Springer Nature Switzerland AG 2020

\begin{abstract}
The problem of unsteady stagnation-point flows of power-law fluids over a porous flat plate with mass transfer is considered with a view to examine the rheological behaviors of the fluids. This study is completely based on the four physical parameters, namely, flow strength parameter $a$, mass transfer parameter $d$, unsteadiness parameter $\beta$ and non-Newtonian power-law index $n$. For $d=0$, the numerical results of this analysis reveal the existence of two types of solutions - one is attached flow solution (AFS) and the other is reverse flow solution (RFS) in a definite range of $n(0<n \leq 2)$ when (a, $\beta)=(1,-1)$. The present analysis confirms that the velocity profile for any dilatant fluid $(n>1)$ matches smoothly with the free stream velocity for a suitable amount of blowing $d(<0)$ depending upon the values of $n$. We will also discuss the asymptotic behaviors of the boundary layer flows for large values of $d$, i.e., for $d \rightarrow \pm \infty$. The asymptotic analysis ensures the existence of the above two solutions for large values of suction $d>0$, whereas the boundary layer solution is terminated after a certain value of blowing $d<0$, dependent on the values of $\beta(<0)$ and $n$. Below this critical value of blowing $d$, this unsteady flow problem also provides us with a solution which does not appear to have a boundary layer character.
\end{abstract}

Keywords Unsteady · Power-law fluids · Porous surface · Dual solutions · Asymptotic solutions

\section{Introduction}

Unsteady boundary layer flows of power-law fluids over flat surfaces represent one of the most classical problems in the practical fluid dynamics. There are numerous avenues in chemical, mechanical, biomedical, mineral and food processing engineering applications where the proper knowledge for settling the flows of non-Newtonian fluids are required to set up the new design techniques in many fluid dynamic devices. Many fluids of practical interest are non-Newtonian and their characteristic features are completely different from that of the viscous Newtonian fluids. However, the use of non-Newtonian fluids have an increasing demand in modern technology as well as in the industrial applications for a number of reasons like drag reduction, modification of effective heat flux, etc, and essentially get a serious attention in advanced and modern fluid dynamics research. There are various models (constitutive equations) available in the open literature to explain the non-Newtonian behaviors of the fluids. Among all the non-Newtonian fluid models, the Ostwald-de-Waele power-law model (Bird et al. [1]) is well accepted since the boundary layer hypotheses can successfully be imposed on this model. Most importantly, this model also describes the behavior of a huge number of real non-Newtonian fluids.

The theoretical research of the steady boundary layer flows for non-Newtonian power-law fluids was first performed by Schowalter [2] and at the same time by Acrivos et al. [3]. Schowalter [2] formulated the boundary layer equations for power-law fluids and also provided the limitations for the existence of the similarity solutions. Acrivos et al. [3] investigated the same problem including the heat transfer analysis. They found that for a dilatant

S. Dholey, sdholey@gmail.com | 'Department of Mathematics, M.U.C. Women's College, Burdwan 713 104, India. 
fluid the boundary layer ends abruptly at a finite distance from the plate surface. This experience has been pointed out as 'finite-width boundary layer' in the literature. On the contrary, for pseudoplastic fluids the stream wise velocity profile matches smoothly with the free stream velocity. An up-to-date literature survey discloses that numerous theoretical works have been done on the boundary layer flows for non-Newtonian fluids over a flat plate surface under various conditions such as Thompson and Snyder $[4,5]$, Teipel [6], Pakdemirli [7], Nachman and Taliaferro [8], Denier and Dabrowski [9], Wel and Al-Ashhab [10] and the references therein. An experimental work on nonNewtonian boundary layer flows over a flat plate is found in a paper by Wu and Thompson [11]. They found that for pseudoplastic fluids, the boundary layer solutions follow the correct behaviour even when the Reynolds number is not large. This is the assumption of the large Reynolds number which reduced the full Navier-Stokes equations into the most simple boundary layer equations.

All the above studies are, however, restricted to the steady boundary layer flows for non-Newtonian fluids, although, the movement of the fluids at the initial stage depends on the time as well. Dholey and Gupta [12] investigated the unsteady two-dimensional separated stagnation-point boundary layer flows for Newtonian fluids over a moving porous surface. A few numbers of studies of the unsteady separated stagnation-point boundary layer flows for Newtonian fluids over a flat plate under various situations are Ma and Hui [13], Dholey [14], Lok and Pop [15] and Dholey [16]. On the contrary, some relevant studies of the unsteady boundary layer flows for non-Newtonian fluids are Xu and Liao [17], Cheng et al. [18], Xu et al. [19] among others. Recently, Dholey [20] investigated the unsteady separated stagnation-point flows of power-law fluids over a moving nonporous surface. The most general formation of the governing unsteady boundary layer equations for non-Newtonian fluids as well as the procedure for achieving the similarity solutions of the same are found in details in this report. However, the effects of the mass transpiration on the unsteady separated stagnationpoint boundary layer flows for power-law fluids over a permeable surface might be huge non-intuitive as well as non-trivial owing to the attracting physics that arises into this flow field by the alteration of the fluid rheology in combination with the mass transfer parameter. Moreover, the consideration of the power-law fluids into the boundary layer flow dynamics gives us an opportunity to investigate the effect of wide spectrum of fluid rheology, for example, shear-thinning fluids $(n<1)$, shear-thickening fluids $(n>1)$ along with Newtonian fluid $(n=1)$ to unveil the essential flow physics of the associated flows.

The main objective of this study is, therefore, to investigate the effects of the mass transfer parameter $d$ on the unsteady separated stagnation-point boundary layer flows for power-law fluids over a permeable surface as well. The unsteadiness in this flow field arises solely from the outer stagnation flow which is inversely proportional to the time $t$. It will be measured by a dimensionless parameter which is known as the unsteadiness parameter $\beta$. A positive value of $\beta$ accelerates the flow (accelerating flow), whereas a negative value opposes (decelerating flow) it. Here, we will show that for all values of $n$ in $(0<n \leq 2)$ and for a given negative value of $\beta(=-1)$, the governing boundary layer equation of this flow problem always provides us with two different types of solutions (AFS and RFS) for any given value of $d \geq 0$, however large it may be. By contrast, the governing boundary layer equation yields only the attached flow solution up to a definite value of $d<0$, which depends highly on the values of $n$. The drag-coefficient, distribution of velocity and the patterns of streamlines have also been presented to elucidate the role of $n$ in this flow dynamics. Interesting enough, the present analysis removes the barrier of the boundary layer solution for a dilatant fluid which has been terminated abruptly at a finite distance from the plate surface by applying a proper amount of blowing velocity owing to the upliftment of the viscous boundary layer. In this paper, we would like to focus on the analysis of the asymptotic behaviors of the boundary layer flows in the limiting cases of the mass transfer $d$ which includes suction $(d>0)$ and blowing $(d<0)$.

We split up this paper into six sections. The mathematical analysis and the essential quantities of physical involvement to this flow problem are presented in Sect. 2. The detailed solution methodology is provided in Sect. 3. Section 4 is committed to the results and discussions where we elaborate the central findings in a comprehensive way. The effects of large mass transfer parameter $d$ on this flow dynamics are discussed in Sect. 5. In Sect. 6, we end up this paper by highlighting some fundamental insights obtained from the present analysis.

\section{Mathematical analysis}

Consider the unsteady two-dimensional stagnation-point boundary layer flows of power-law fluids over a permeable surface coinciding with the plane $y=0$. We choose the Cartesian coordinates $(x, y)$ such as the $x$-axis is taken along the permeable surface and the $y$-axis is normal to it. The corresponding velocity components and time are represented by $(u, v)$ and $t$, respectively. Since the flow is unsteady, the outer inviscid flow as well as the suction (or blowing) velocity must be the functions of space $x$ and time $t$. We assume these velocities as $u_{e}(x, t)$ and $v_{w}(x, t)$, respectively. 
The rheological equation of state for non-Newtonian fluids which obeys the Ostwald-de Waele power-law model (Bird et al. [1]) is defined by

$\tau_{i j}=2 K\left(2 D_{l m} D_{l m}\right)^{(n-1) / 2} D_{i j}$.

Here $\tau_{i j}$ is the deviatoric part of the stress tensor $\sigma_{i j}=-p \delta_{i j}+\tau_{i j}$, where $p$ denotes the fluid pressure. The rate-of-strain tensor is given by

$D_{i j}=\frac{1}{2}\left(\frac{\partial u_{i}}{\partial x_{j}}+\frac{\partial u_{j}}{\partial x_{i}}\right)$

Both $K$ and $n$ are taken as positive and they represent the consistency coefficient and the power-law (viscosity) index, respectively. For $n=1$, the constitutive Eq. (1) reduces to the corresponding expression of Newtonian fluids with dynamic coefficient of viscosity $\mu(=K)$. The fluids are called the shear-thinning (pseudoplastic) fluids when $n<1$, whereas the shear-thickening (dilatant) fluids when $n>1$.

One can rationalize the unsteady Navier-Stokes equations for non-Newtonian fluids (following Ostwald-de Waele power-law model) by using the Prandtl's boundary layer hypotheses. After this, he gets the governing equations for this flow problem in dimensionless form as (Dholey [20])

$\frac{\partial u}{\partial x}+\frac{\partial v}{\partial y}=0$

$\frac{\partial u}{\partial t}+u \frac{\partial u}{\partial x}+v \frac{\partial u}{\partial y}=\frac{\partial u_{e}}{\partial t}+u_{e} \frac{\partial u_{e}}{\partial x}+\frac{\partial}{\partial y}\left(\left|\frac{\partial u}{\partial y}\right|^{n-1} \frac{\partial u}{\partial y}\right)$

where the external flow velocity $u_{e}(x, t)$ relates to the pressure $p$ by the following relation

$\frac{\partial u_{e}}{\partial t}+u_{e} \frac{\partial u_{e}}{\partial x}=-\frac{\partial p}{\partial x}$.

The boundary conditions corresponding to the Eqs. (3) and (4) are

$u(x, 0, t)=0, v(x, 0, t)=v_{w}(x, t) \quad$ and

$u(x, y, t) \rightarrow u_{e}(x, t) \quad$ as $\quad y \rightarrow \infty$.

We presume the dimensionless stream function $\psi(x, \eta, \tau)$ and the similarity variable $\eta$ as proposed by Dholey [20] are:

$\psi=a \frac{x^{\frac{2 n}{1+n}}}{\tau^{\frac{2 n-1}{1+n}}} f(\eta) \quad$ and $\quad \eta=\frac{x^{\frac{1-n}{1+n}}}{\tau^{\frac{2-n}{1+n}}} y$,

where $a(>0)$ is the flow strength parameter, $\tau\left(=t_{\text {ref }}-\beta t>0\right)$ is the transformation of dimensionless time $t$ and $t_{\text {ref }}$ is the initial reference value of $t$. The similarity solutions of Eq. (7) routinely satisfy the continuity Eq. (3). Now the dimensionless velocity components $u$ and $v$ in term of the similarity function $f(\eta)$ can be obtained from (7) as follows:

$$
\begin{aligned}
u & =\frac{\partial \psi}{\partial y}=a \frac{x}{\tau} f^{\prime}(\eta) \quad \text { and } \quad v=-\frac{\partial \psi}{\partial x} \\
& =-a \frac{x^{\frac{n-1}{n+1}}}{\tau^{\frac{2 n-1}{n+1}}}\left[\left(\frac{2 n}{n+1}\right) f(\eta)+\left(\frac{1-n}{1+n}\right) \eta f^{\prime}(\eta)\right] .
\end{aligned}
$$

Here prime denotes differentiation with respect to $\eta$. The vertical component of velocity $v$ in (8) recommends that the suction (or blowing) velocity $v_{w}(x, t)$ will be in the form given as below:

$v_{w}(x, t)=-a \frac{x^{\frac{n-1}{n+1}}}{\tau^{\frac{2 n-1}{n+1}}}\left(\frac{2 n}{n+1}\right) d$,

where $d(=f(0))$ is a non-dimensional quantity measures the effectiveness of the mass transpiration rate such as suction or injection (blowing) accordingly as $d>$ or $<0$.

The external flow velocity is characterized by the stream function

$\psi_{e}=a \frac{x y}{\tau}$,

with the corresponding components of velocity given as below:

$u_{e}=a \frac{x}{\tau} \quad$ and $\quad v_{e}=-a \frac{y}{\tau}$.

Substituting (8) into (4), one can obtain the governing boundary layer equation as

$$
\begin{aligned}
& a^{n-1}\left(\left|f^{\prime \prime}\right|^{n-1} f^{\prime \prime}\right)^{\prime}+a\left(\frac{2 n}{n+1}\right) f f^{\prime \prime}-a f^{\prime 2} \\
& \quad+a-\beta\left[\left(\frac{2-n}{1+n}\right) \eta f^{\prime \prime}+f^{\prime}-1\right]=0 .
\end{aligned}
$$

The boundary conditions (6) by the use of (8)-(11) as

$f(0)=d, f^{\prime}(0)=0 \quad$ and $\quad f^{\prime}(\eta) \rightarrow 1$ as $\eta \rightarrow \infty$.

For $d=0$, Eqs. (12) and (13) corroborate with the equations (39) and (40) of Dholey [20]. Also for $n=1$ and $d=0$, Eqs. (12) and (13) coincide with the Eqs. (10) and (11) of Dholey [14]. Again for $a=n=1$ and $d=\beta=0$, Eqs. (12) and (13) become the classical equations of the steady stagnation-point boundary layer flow of a viscous fluid over an infinite plane wall as presented by Hiemenz [21].

\subsection{Physical quantities of interest}

In practical fluid dynamics, the prime motive for the application of injection is to reduce the drag. On the contrary, 
suction tends to thin the thickness of the layer for which the wall gradient is increased. As a result, the flow separation inside the layer is suppressed by reducing the possibility of having vanishing wall shear stress. Therefore, the main focus of the present study is to show the variation of drag as a function of the fluid rheology $n$ in combination with the mass transfer parameter $d$. For these reasons, we have considered the following two important physical quantities:

(i) Drag-coefficient:The dimensionless drag-coefficient $C_{D}$ on the plate surface of length / and unit breadth is defined by

$C_{D}=\frac{2}{\rho U_{0}^{2} l} \int_{0}^{l} \tau_{x y} d x=\frac{2 \mu_{0}}{\rho U_{0}^{2} l} \int_{0}^{l}\left(\left|\frac{\partial u(x, 0, t)}{\partial y}\right|^{n-1} \frac{\partial u(x, 0, t)}{\partial y}\right) d x$,

where $\mu_{0}$ is the dimensionless form of the consistency coefficient $(K)$ and $U_{0}$ is the velocity scale. Substituting the dimensionless similarity variable $\eta$ and $f$, and executing the integration results as
Eq. (16) indicates that a suitable amount of suction $d(>0)$, dependent on the values of $a, n$ and $\beta$, can remove the displacement thickness by removing the low energy fluid over the plate surface. After this suction value, a reversal of the displacement effect must arise within the flow (see Fig. 4).

\section{Numerical method}

The governing non-linear Eq. (12) subjected to (13) has been solved numerically by employing the fourth-order Runge-Kutta method modified by Gill (Ralston and Wilf [22]). This method gives us accurate results for pseudoplastic as well as Newtonian fluids $(n \leq 1)$, while for dilatant fluids $(n>1)$ the solution does not follow the correct asymptotic behavior owing to the discontinuities in thirdand higher-order derivatives of $f$ at a certain value $\eta_{c}$ of $\eta$ (Teipel [6]). As a result, the boundary layer is terminated abruptly at a finite distance $\eta=\eta_{c}$ from the bounding sur-

$$
\begin{aligned}
C_{D} & =\frac{2}{\operatorname{Re}^{1 /(n+1)}} \frac{(n+1)}{(3 n+1)} \frac{a^{n}}{\tau^{3 n /(n+1)}}\left(f^{\prime \prime}(0)\right)^{n} \text { when } \frac{\partial u(x, 0, t)}{\partial y}>0 \\
& =-\frac{2}{\operatorname{Re}^{1 /(n+1)}} \frac{(n+1)}{(3 n+1)} \frac{a^{n}}{\tau^{3 n /(n+1)}}\left(f^{\prime \prime}(0)\right)^{n} \text { when } \frac{\partial u(x, 0, t)}{\partial y}<0 .
\end{aligned}
$$

Here $\operatorname{Re}\left(=\rho U_{0}^{2-n} /^{n} / \mu_{0}\right)$ denotes the Reynolds number which is compatible with this flow problem. Equation (15) shows that $C_{D}$ is directly proportional to the $n$-th power of the wall velocity gradient. Indeed, the values of $\left(f^{\prime \prime}(0)\right)^{n}$ are needed to estimate the drag-coefficient. Thus, to show the variation of $\left(f^{\prime \prime}(0)\right)^{n}$ against $n$ for dissimilar values of $d$ in combination with $\beta$ is another important part of the paper which is given in Sect. 4 .

(ii) Displacement thickness: Owing to the viscosity, fluid velocity adjoining the plate surface is less than the external flow velocity. The reduction in the total flow rate caused by this action is given by $\int_{0}^{\infty}\left(u_{e}-u\right) d y$. In fact, the displacement thickness is the vertical height measured from the bounding surface by which the external flow is shifted on account of the boundary layer. Therefore, the order of the displacement thickness will be the same as that of $y$. Hence for displacement thickness $\delta$, we have

$$
\begin{aligned}
\frac{a}{\tau} \delta & =\frac{d}{d x} \int_{0}^{\infty}\left(u_{e}-u\right) d y \\
\Rightarrow \bar{\delta} & =\frac{x^{\frac{1-n}{n+1}}}{\tau^{\frac{2-n}{n+1}}} \delta=\left(\frac{2 n}{n+1}\right)\left[\lim _{\eta \rightarrow \infty}(\eta-f(\eta))+d\right] \\
\Rightarrow \lim _{\eta \rightarrow \infty}(\eta-f(\eta)) & =\left(\frac{n+1}{2 n}\right) \bar{\delta}-d .
\end{aligned}
$$

Equation (16) ensures that $\bar{\delta}$ is free from $x$ when $n=1$, while for $n \neq 1$ it depends highly on the values of $x$. Besides this, face depending upon the values of $n(>1)$. A secondary viscous adjustment layer is necessary for correct matching of the solution with the free boundary condition (Denier and Dabrowski [9]). It is not the problem in the current study since the contents of the present analysis are completely different from that by Denier and Dabrowski [9]. However, following Teipel [6], we will change the outer boundary condition of (13) into the following forms:

$f^{\prime}=1, \quad f^{\prime \prime}=0 \quad$ for $\quad \eta \geq \eta_{c^{\prime}}$

which will remove the above discontinuity and become suitable for achieving the solutions of this flow problem when $n>1$. During the numerical computations, the step size is taken as $d \eta=0.01$ and the results are corrected within a tolerance level of $10^{-6}$.

For $\beta<0$, the numerical computations reveal two categories of solutions - attached flow solution (AFS) and reverse flow solution (RFS). In particular, when $d=0$ and for the pair of values $(a=1, \beta=-1)$, the present flow problem provides us dual solutions: one AFS and other is RFS for all values of $n$ considered. For conciseness of this paper, we will consider only the above pair of values of $(a$, $\beta$ ) throughout the analysis unless stated otherwise, and demonstrate the effects of the mass transfer parameter $d$ on all types of fluid rheology encircling pseudoplastic fluids $(n<1)$, Newtonian fluids $(n=1)$ and dilatant fluids $(n>1)$. 


\section{Results and discussion}

The ongoing analysis is completely a theoretical one and depends on the four physical parameters, namely: (i) flow strength parameter $a$, (ii) suction/blowing parameter $d$, (iii) power-law index $n$ and (iv) unsteadiness parameter $\beta$. Here, we have presented only the central findings of this analysis for some values of these parameters in graphical forms from which one can easily estimate the flow behaviors in other values of these parameters.

\subsection{Effects of suction/blowing on the drag-coefficient}

We begin our discussion with Fig. 1, which conveys the information about the wall velocity gradient $f^{\prime \prime}(0)$ obtained as a function of the power-law (viscosity) index $n$ for three different values of $d(=-0.25,0.0$ and 0.25$)$ in cases of both AFS and RFS flows. This figure demonstrates that for any given value of $d$, irrespective of its sign, the magnitude values of the wall velocity gradient decrease gradually with an increasing value of $n$ for both types of flows. In fact, the boundary layer thickness reduces as the viscosity index $n$ increases. This phenomenon is accredited to the increase in the non-Newtonian viscosity of the shear-thickening fluids caused by the decrease in the shear rate. It is noticeable that for an impermeable wall (i.e., for $d=0$ ), the values of the velocity gradient $f^{\prime \prime}(0)$ for both flows approach asymptotically to two different constant values, negative for RFS flow and positive for AFS flow, with an increasing value of $n$. This result confirms the existence of dual solutions (one AFS and the other is

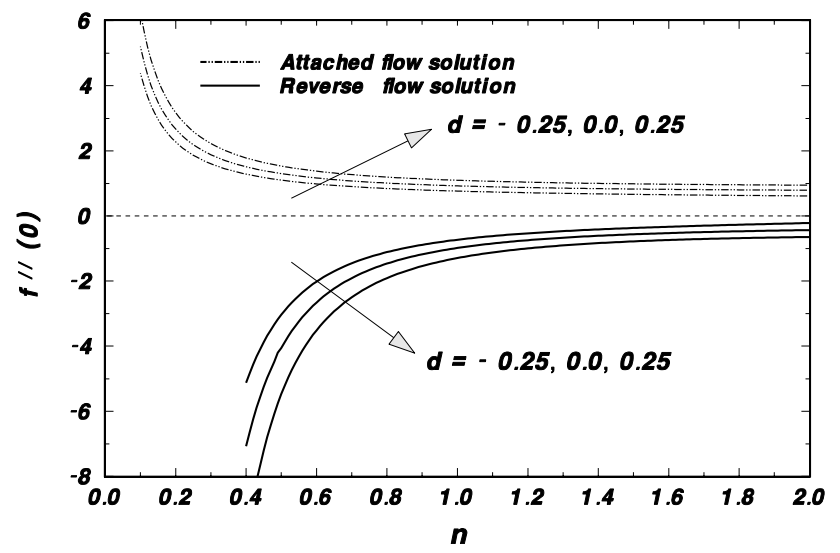

Fig. 1 Wall velocity gradient $f^{\prime \prime}(0)$ curves as a function of $n$ for three dissimilar values of $d(=-0.25,0.0$ and 0.25$)$ in cases of both AFS and RFS. The magnitude values of $f^{\prime \prime}(0)$ increase with an increase of suction $d$, whereas they decrease with blowing $d$. This result is more pronounced for pseudoplastic fluids $(n<1)$, although it is true for all types of fluids
RFS) of this flow problem for all types of fluid rheology $n(0<n \leq 2)$ in case of a nonporous plate, i.e., for $d=0$.

The above result is true for all values of suction $d>0$, whereas it does not hold true for large rate of blowing $d<0$, dependent on the values of $n$. It is obvious that for both types of flows as well as for all kinds of fluids (i.e., for all values of $n)$, the magnitude values of $f^{\prime \prime}(0)$ increase with an increasing value of suction $d$ relative to those values for $d=0$. This result is more pronounced in shear-thinning fluids $(n<1)$ than for shear-thickening fluids $(n>1)$ in comparison with the Newtonian viscous fluids $(n=1)$. This, however, establishes the fact that an application of suction increases the wall velocity gradient following the suppression of the boundary layer by removing the non zero vorticity layer (lowest energy fluids) which originates nearest to the plate surface owing to the frictional forces. Interesting enough, the magnitude of $f^{\prime \prime}(0)$, especially for RFS flow, increases drastically with an increase of suction $d$. This phenomenon suggests that for a negative value of $\beta(=-1)$ the reverse flow solution of this flow problem will continue for large rate of suction $d$, whatever be the rheological nature of the fluids (see Fig. 8).

On the other hand, the reverse scenario is found in case of blowing so as the magnitude values of $f^{\prime \prime}(0)$ decrease with an increase of blowing $|d|$ when $d<0$, for both types of flows even for all kinds of fluids. This result confirms that after a certain value of blowing $d$, dependent on $n$, the wall velocity gradient becomes zero for both flows when $(a+\beta)=0$ (see Fig. 9). Most importantly, for a given value of $n$ the magnitude value of blowing is comparative less for RFS flow than for AFS flow in order to vanish the wall velocity gradient. This observation, which led the authors Dholey and Gupta [12] to come to this conclusion that after a certain value of blowing $d(\approx-4.5)$, only the AFS exists and the solution becomes unique. However, this result is not true for large rate of blowing $d$, which we will discuss in Sect. 5, where we will also show that the solution of the current flow problem does not continue after a definite value of blowing $d$ depending upon the values of $n$ when $(a+\beta)=0$ which was considered by the authors of Ref. [12] in their study. According to Fig. 1, for pseudoplastic fluids, the magnitude value of blowing required for obtaining the zero wall velocity gradient will relatively be higher than that of dilatant fluids (see also Fig. 9). This phenomenon ensures that for any given value of $d$, disregarding its sign, the solution domain of the similarity function $f$ will continuously be decreased with an increasing value of $n(0<n \leq 2)$. Finally, we can conclude that the present flow problem yields only a unique attached flow solution up to a certain critical value of blowing $d$ depending upon the values of $n$ as well as on the sum values of $(a+\beta) \geq 0$. Below this critical value of blowing $d$, no solution will exist for this flow problem (see Fig. 9). 


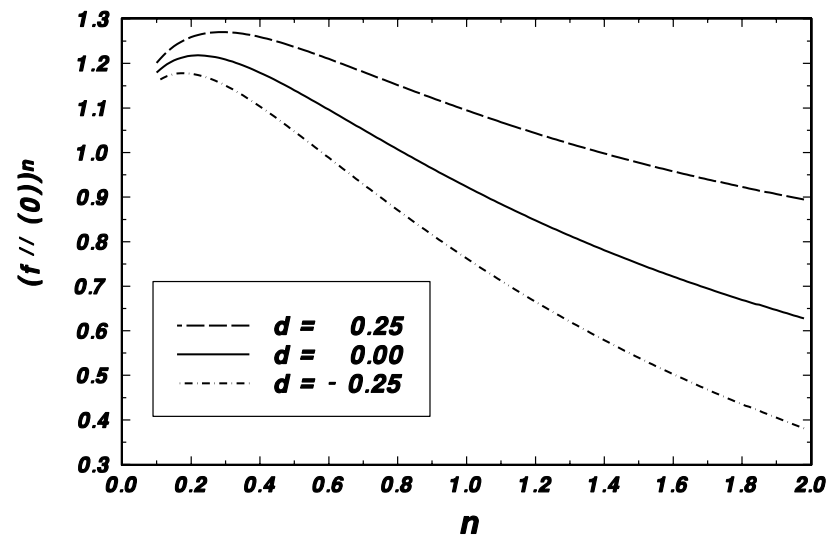

Fig. 2 Change of drag-coefficient $\left(f^{\prime \prime}(0)\right)^{n}$ as a function of $n$ for three dissimilar values of $d(=-0.25,0$ and 0.25$)$ in case of AFS only. A small amount of blowing can reduce a sufficient amount of dragcoefficient and it is significantly effective in higher values of $n$

Figure 2 shows the change of drag-coefficient $\left(f^{\prime \prime}(0)\right)^{n}$ against $n$ for various values of $d$, indicated on the figure, for AFS flow only. It is readily seen that for a given value of $n$, the friction drag-coefficient continuously decreases with an increasing value of $|d|$ when $d<0$. This phenomenon is in accordance with the physical situation that an increase of blowing of the fluid amounts to the flow system which is akin to the increase of the vertical component of velocity resulting in the decrease of the frictional force at the plate surface. The opposite result is true for suction imposed at the wall. It can also be seen that for a given value of $d$ the drag-coefficient decreases consistently with the increase of $n$ ( $\approx 0.2$ and above), and the rate of decrease is higher for high dilatant fluids. This is because of the fact that relatively lesser wall velocity gradient is instinctive to the class of shear-thickening (dilatant) fluids which essentially reduces the friction drag-coefficient appreciably by an increasing value of $n$. However, from this figure one can obtain the reduced amount of drag-coefficient in percentage for a small value of blowing $d=-0.25$ (relative to zero mass transfer $(d=0)$ at the wall) are $4.84,8.11,11.64$, $17.42,21.55,28.11$ and 35.03 corresponding to the values of $n=0.3,0.5,0.7,1.0,1.2,1.5$ and 1.8 , respectively. Hence, we can conclude that the smaller drag-coefficient can be obtained either by applying a greater amount of blowing at the wall or by using a highly shear-thickening fluid or both into the flow system.

\subsection{Effects of suction/blowing on the similarity profiles}

The influence of the mass transfer parameter $d$ on the similarity profiles $f^{\prime}(\eta)$ is exhibited in Fig. 3a-c for three different values of $n$, encircling a dilatant fluid $(n=1.5)$, a pseudoplastic fluid ( $n=0.5)$ along with the special case of Newtonian fluid $(n=1)$ in cases of both AFS and RFS flows. Here, we have included the Fig. 3a only to highlight the change of the unsteady boundary layer flow dynamics of non-Newtonian fluids $(n \neq 1)$ from that of Newtonian fluids $(n=1)$ as well. The horizontal component of velocity $f^{\prime}(\eta)$ at a given location increases with an increasing value of suction $d$, whereas it decreases with an increase of blowing $|d|$ when $d<0$, in case of AFS flow and for all types of fluids $(0<n \leq 2)$. This is because of the fact that increase in suction makes the layer thinner which in turn leads to the increase of the wall velocity gradient resulting in the increase of the velocity inside the boundary layer. The reverse explication holds true in case of blowing given at the wall. A closer look at Fig. $3 \mathrm{~b}$ discloses the fact that for blowing $d(<0)$, the boundary layer solution captures the correct asymptotic behavior, whereas for a given value of $d(\geq 0)$ it is terminated after a certain distance (for example $\eta=\eta_{c}$ ) from the porous bounding surface depending upon the values of $n>1$. In fact, for blowing the thickness of the boundary layer is increased with a concomitant decrease in the wall velocity gradient. Thus, we see that the boundary layer solution of a shear-thickening fluid which is terminated at a finite distance from the plate surface will now match correctly with the outer boundary condition for a suitable amount of blowing $d$ depending upon the values of the other parameters $a, \beta$ and $n>1$.

On the contrary, specific to the case of RFS flow and for all types of fluids, the velocity profiles $f^{\prime}(\eta)$ nearer to the surface are accredited to the flow reversal phenomena, albeit they become positive far away from the plate surface to retrieve the free boundary condition. As a result, a minimum velocity is occurred at a point on the reverse flow profile where the velocity gradient is zero. It is found that the minimum velocity increases with the increase in suction owing to the decrease of $f^{\prime \prime}(0)$ which is negative for RFS flow. This phenomenon suggests the existence of reverse flow solution for large value of suction $d$, which is shown in Fig. 8. On the other hand, the velocity profile $f^{\prime}(\eta)$ approaches zero with the increase of blowing $d$ since $f^{\prime \prime}(0)$ tends to zero for large values of blowing $d$ depending upon the values of $n$ (see Fig. 9). However, the minimum velocity continues to go by the pulling forces of the comparatively higher viscosity of the pseudoplastic fluids up to a certain distance perpendicular to the plate surface and ultimately $f^{\prime}(\eta)$ changes its direction to retrieve the far-field boundary condition. As a consequence, a plateau region is manifested in the neighborhood of this minima and the stretch of the plateau region decreases with the increase of suction $d$. These results are shown in Fig. 3c. On the contrary, Fig. $3 \mathrm{~b}$ clearly demonstrates that for a dilatant fluid the minimum velocity appears in the velocity profile with a sharp peak. It can also be seen that the 


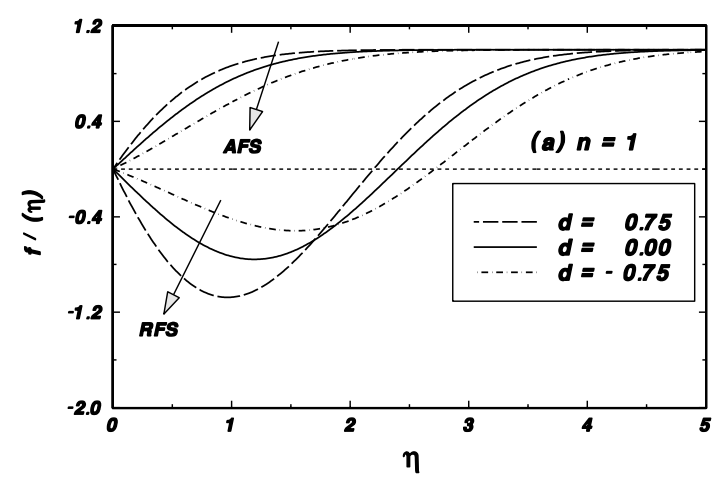

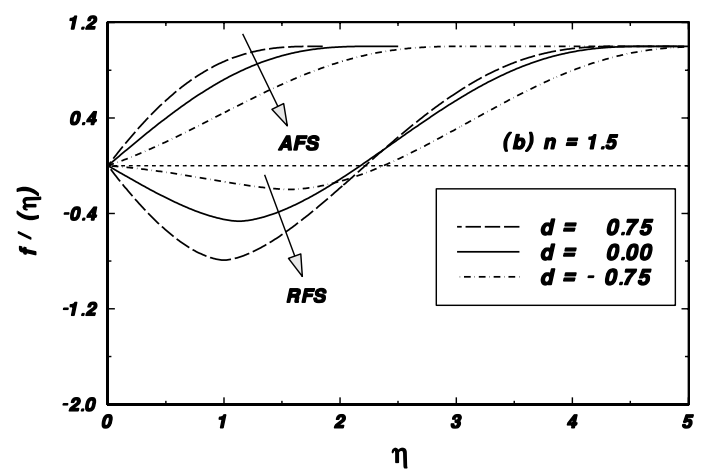

Fig. 3 Similarity profiles $f^{\prime}(\eta)$ against $\eta$ in cases of both AFS and RFS flows for three different values of $d(=-0.75,0.0$ and 0.75$)$ when: (a) $n=1.0$, (b) $n=1.5$ and (c) $n=0.5$. For a dilatant fluid, the attached flow solution is ended at a finite distance from the porous bounding surface when $d \geq 0$, while it matches smoothly with the far-

location of this minimum velocity comes closer to the plate surface with an increasing value of suction $d$ that essentially makes the boundary layer thinner. This result is more effective for pseudoplastic fluids than the dilatant fluids in comparison with the Newtonian fluids (see also Fig. 8). This is because of the fact that for the same values of $a, d$ and $\beta$, the wall velocity gradient which is a strong function of the fluid rheology is different for different values of $n$.

We have pointed out earlier that the displacement thickness $\bar{\delta}$ can be removed by applying a proper amount of suction $d$ depending upon the rheological behavior of the fluids since it is directly connected to the parameters $d$ and $n$ (see Eq. (16)). In order to validate this result, we have depicted the Fig.4a-c which demonstrate the impact of the mass transfer parameter $d$ on the variation of $f(\eta)$ against $\eta$ for three different values of $n$ which covers all kinds of fluids. From these figures it is clear that the vertical component of velocity $f(\eta)$ increases with the increase

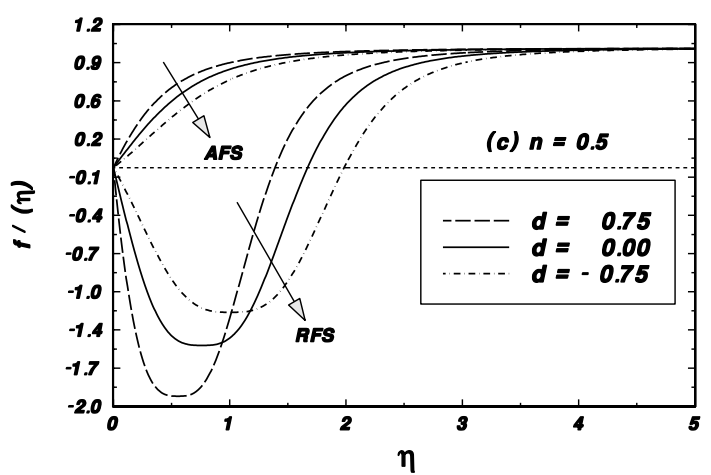

field boundary condition for blowing $d(<0)$. For a pseudoplastic fluid, the reversae flow solution makes a plateau region in the vicinity of the minimum velocity owing to the higher viscosity of this kind of fluid, whereas a sharp peak is found there for a dilatant fluid

of suction $d$, whereas it decreases with blowing $d$. As a consequence, the boundary layer displacement thickness $\bar{\delta}$ decreases continuously with suction $d$ and finally it becomes vanish at a definite value of $d$ (say, $d_{s}$ ) depending upon the values of $n$. Here, we have found numerically that the zero-displacement-thickness suction values are $d_{s}=0.52616,0.55472$ and 0.55640 corresponding to the values of $n=0.5,1.0$ and 1.5, respectively. Figure 4a which is depicted for $n=1$, authenticated our present numerical results $\left(\bar{\delta}\right.$ and $\left.d_{s}\right)$ with the corresponding results reported in Ref. [12] (see Table II there). Besides this, these figures indicate that after this suction value of $d_{s}$ a reversal of the displacement effect must arise within the flow. Finally, we see that as the value of $n$ increases, more suction is needed to obtain the zero-displacement-thickness velocity profile inside the layer. In addition to this, it is found that the value of $d_{s}$ depends weakly on the shear-thickening fluids (i.e., for $n>1$ ). 


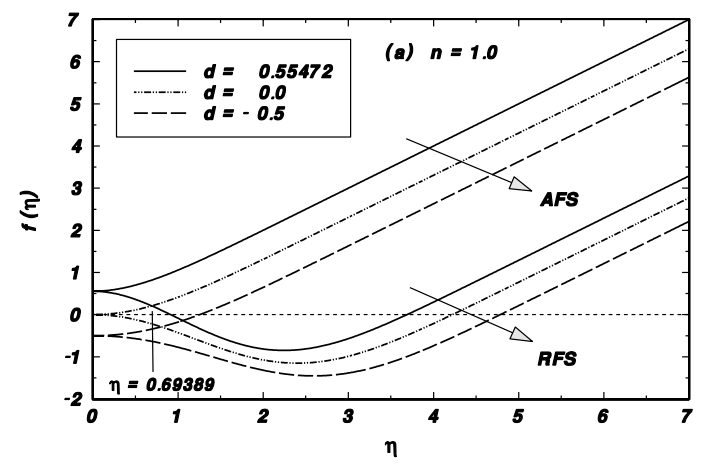

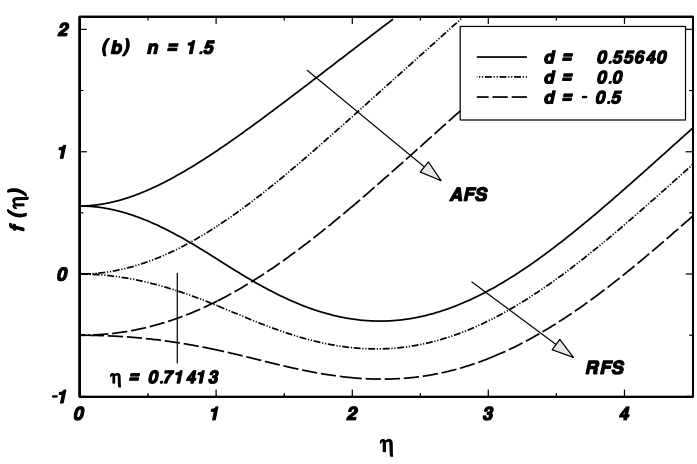

Fig. 4 Similarity profiles $f(\eta)$ against $\eta$ in cases of both AFS and RFS flows for three different values of $d$ when: (a) $n=1$, (b) $n=1.5$ and (c) $n=0.5$. The vertical component of velocity $f(\eta)$ increases with a concomitant decrease of the boundary layer thickness in an

\subsection{Effects of suction/blowing on the streamlines patterns}

The stability of the boundary layer flows is significantly affected by the occurrence of stagnation-points inside the layer since separation appears to start from the stagnation-points (Rosenhead [23]). Hence the detection of the positions of the stagnation-points within the viscous layer for different types of fluids rheology (i.e., for different values of $n$ ) is another important part of this analysis. However, the positions of the stagnation-points $\left(x_{s}, \eta_{s}\right)$ inside the layer will be obtained from (8) by setting $u=v=0$ and is given by

$x_{s}=0 \quad$ and $\quad y_{s}=\tau^{\frac{2-n}{1+n}} X^{\frac{n-1}{n+1}} \eta_{s^{\prime}}$

where $\eta_{s}$ satisfies

$2 n f\left(\eta_{s}\right)+(1-n) \eta_{s} f^{\prime}\left(\eta_{s}\right)=0$.

Figure5a-e display the patterns of streamlines for three different values of $n(=0.5,1.0$ and 1.5$)$ along with the suction and injection values of $d(= \pm 1,0, \pm 0.5)$ in case of RFS flow only. Here, we have placed the streamlines for the

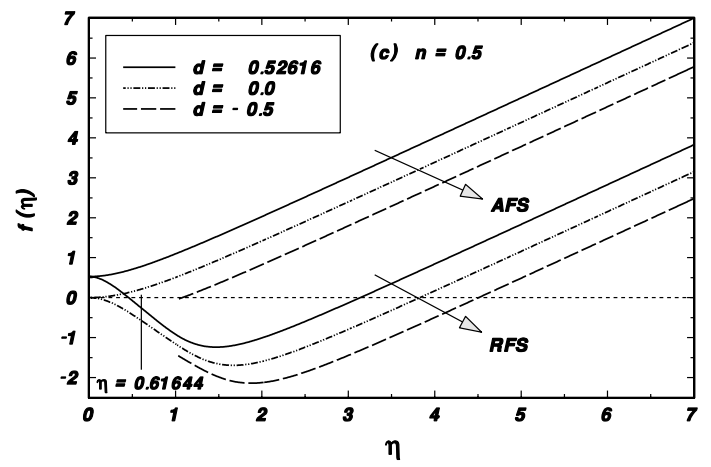

increase of suction $d$ and ultimately it becomes zero for a definite value of suction $d$ depending upon the values of $n$. Here, the curves for $d>0$ are the zero-displacement-thickness velocity profiles corresponding to the values of $n$ as labelled in each figure

above three values of $n$ in a given $d$ are side after side from which one can easily appreciate the flow dynamics of all types of fluids rheology. These figures are the evidence of the existence of the non zero stagnation-point(s) within the flow in a given value of $d$, whatever be its sign. Two non zero stagnation-points are found for suction, while only one non zero stagnation-point exists inside the layer either for zero mass transfer or for blowing given at the wall. Indeed, non zero stagnation-point originates inside the layer only when the vertical component of velocity $f(\eta)$ has a reverse flow zone and simultaneously it crosses the separating streamline $\psi=0$. As the flow is reverse, $f(\eta)$ has a reverse flow zone inherently for zero mass transfer $(d=0)$ at the wall and we have found only one non zero stagnation-point, no matter what type of fluid is considered in this case (see Figs. 4 and $5 \mathrm{c}$ ).

Interesting enough, suction $(d>0)$ increases (lifts-up) the velocity profile $f(\eta)$ for which it crosses the separating streamline twice, resulting in the existence of two non zero stagnation-points within the flow (see Figs. 4, 5a and b). The locations of these stagnation-points come closer to each other with an increasing value of suction $d$ and finally they merge with one another at a definite value of suction $d$ that depends on the values of $n$. After this suction value 

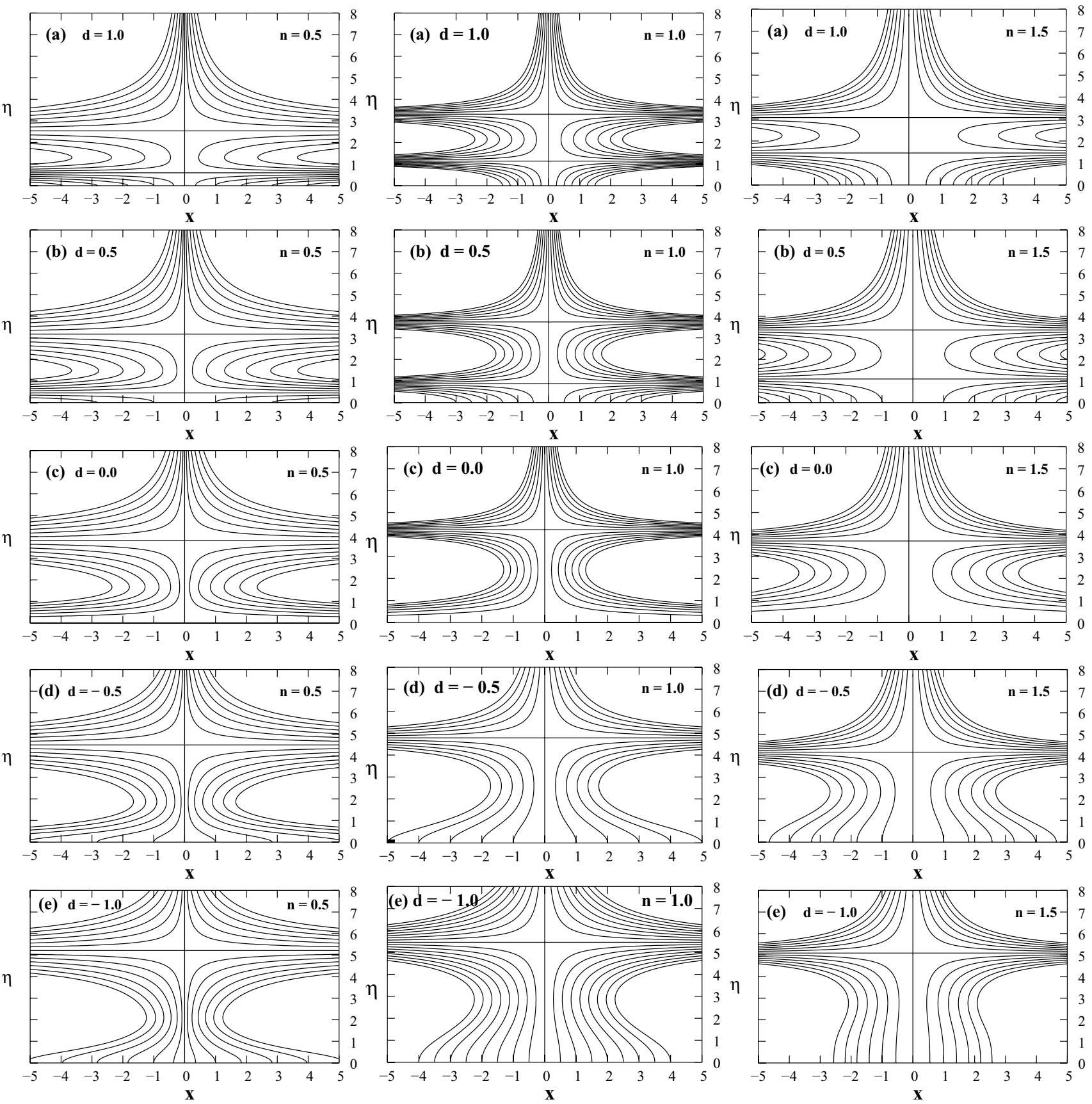

Fig. 5 (a)-(e) Contour plots of the stream function $\psi$ in case of RFS flow for various values of $d$ and $n$ when $\tau=1$. The values of the streamlines are : $-1.2(0.2) 1.2$. The streamlines for pseudoplastic fluids are closer to the separated streamline $\psi=0$ than that of the dilatant fluids. When $d>0$, two non-zero stagnation-points are found within the flow while for $d \leq 0$, only one non-zero stagnation-point is manifested there

stagnation-point within the flow, no matter what type of fluid is considered in this case (see Figs. $4,5 \mathrm{~d}$ and e). The location of this stagnation-point moves further and further away from the plate surface $(\eta=0)$ with an increasing value of blowing $d$ and ultimately after a certain value of blowing $d$, dependent on $n$, the velocity profile $f(\eta)$ can not cross the separating streamline owing to the blowing streamline once resulting is the existence of one non zero 
off of the separating streamline. Consequently, the stagnation-point boundary layer flow solution ends after this value of blowing $d$ (see Fig. 9). Indeed, these kinds of flow behaviors essentially stem from the fact that suction tries to lifts-up the boundary layer flow thereby two different non-zero stagnation-points come closer to each other, while blowing at the wall makes an effort to depress the flow which in essence pushes away the stagnation-point from the plate surface.

The influence of the fluid rheology on this flow dynamics is reflected by the alteration of the locations of onset stagnation-points within the flow with or without mass transfer at the wall. For a given value of suction $d$, the locations of the stagnation-points are at larger distances from the plate surface as observed in case of dilatant fluids essentially attributable to the less velocity gradient at the wall (see Fig. 5a and b). An opposite result is found for blowing $d$ (see Fig. $5 d$ and e). A careful scrutiny at these contour plots discloses the fact that for any given value of $d$ considered, the streamlines are closer to the separating streamline $\psi=0$ for pseudoplastic fluids than that of the dilatant fluids relative to viscous fluids. This result is in accordance with the fact that an increase in the nonNewtonian behavior of the fluid implies the decrease in the shearing motion which in turn leads to the movement of the other streamlines apart from the separating streamline.

In contrast with the streamline patterns as shown in Fig. $5 \mathrm{a}$ and $\mathrm{b}$ for suction $d$, where two non-zero stagnation-points exist inside the layer, here, for AFS flow, no non-zero stagnation-point exists for $d \geq 0$. But remarkably, like RFS flow, the contour plots of the stream function in case of AFS flow for blowing $d(<0)$ conceives only one non zero stagnation-point as the velocity profile $f(\eta)$ crosses the separating streamline once in this case (see Fig. 4). This fact is clearly exhibited in Fig. 6a and b which are delineated with the same values of blowing $d$ and viscosity index $n$ as considered for the Fig. $5 \mathrm{~d}$ and e. The novelty which arises from Fig. 6 is that for a given value of blowing $d$, the height of the stagnation-point from the plate surface increases with an increasing value of $n$. Besides this, increase of blowing velocity allows the stagnation-point to move away from the plate surface owing to the upliftment of the separating streamline. A comparative study between the Figs. 5 and 6 for any given values of blowing $d$ and $n$ divulges that the existing position of the non zero stagnation-point for RFS flow is always higher than that of AFS flow. The underlying physics behind such position of the stagnation-point is directly related with the attribution of the velocity profiles $f(\eta)$ for both AFS and RFS flows (see Fig. 4). Finally, we can conclude from Figs. 5 and 6 that the flows are separated for all types of fluids even for all values of $d$ considered, as the streamlines emerge from the plate surface.
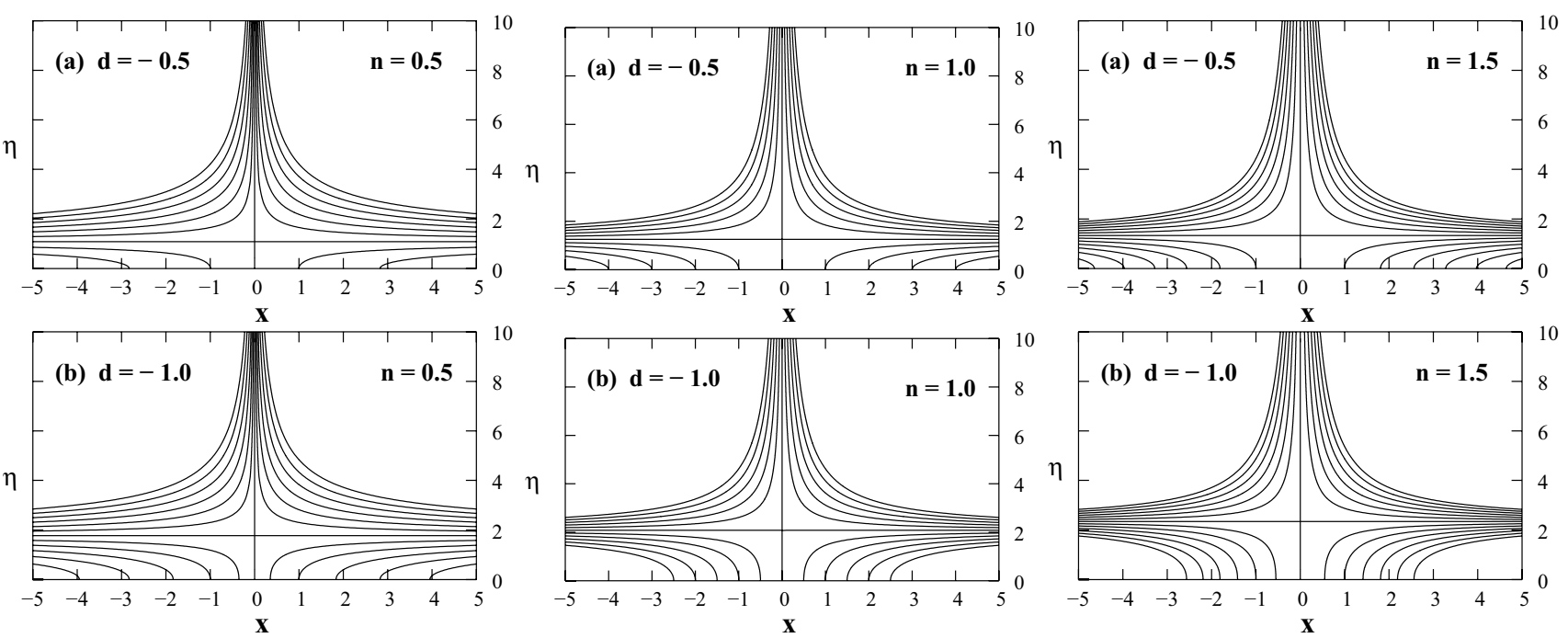

Fig. 6 (a)-(b) Contour plots of the stream function $\psi$ in case of AFS flow for various values of blowing $d$ and viscosity index $n$ when $\tau=1$. Here, we have used the same values of the streamlines as considered in Fig. 5. Streamlines of the attached flow solution con- ceives one and only one non zero stagnation-point within the flow only in case of blowing. This stagnation-point moves away from the plate surface with the increase of blowing $d$ as well as the increase of the viscosity index $n$ 


\section{Asymptotic analysis}

We have already examined, in detail, the influence of the mass transfer parameter $d$ on the boundary layer flows of power-law fluids over a porous flat surface with associated physics. The foregoing analysis suggests that the solutions of this flow problem discussed until now can easily be extended to the limiting cases of strong suction $(d \gg 1)$ as well as massive blowing $(d \ll-1)$ imposed at the wall. In this section, we will show that the boundary layer solution of this flow problem does not continue for large rate of blowing $d$, while they exist for large rate of suction $d$. In addition to this, we will show numerically that for large value of blowing $d$, the attached flow solution continues up to a definite value of $\beta(<0)$ that depends on the sum values of $(a+\beta)$ which is positive.

\subsection{Effects of strong suction $(d \gg 1)$}

Here, we make an attempt to show the impact of strong suction (i.e., $d \rightarrow \infty$ ) on the boundary layer flows of power-law fluids over a porous flat surface as well. For this purpose, we introduce a new function $F(\omega)$ using the similar logic as found in the work of Schlichting and Gersten [24]:

$f(\eta)=d+\frac{1}{d^{\frac{1}{n}}} F(\omega) \quad$ with $\quad \omega=\eta d^{\frac{1}{n}}$.

Substituting (20) into (12) and (13) and taking $d \rightarrow \infty$, one can get the governing boundary layer equation for large rate of suction as:

$\left(\left|F^{\prime \prime}\right|^{n-1} F^{\prime \prime}\right)^{\prime}+a^{2-n}\left(\frac{2 n}{n+1}\right) F^{\prime \prime}=0$,

$F(0)=0, F^{\prime}(0)=0 \quad$ and $\quad F^{\prime}(\omega) \rightarrow 1$ as $\omega \rightarrow \infty$.

Here prime denotes the derivative with respect to the stretched variable $\omega$. It is noticeable that the Eq. (21) is free from the unsteadiness parameter $\beta$ since $1 / d^{(n+1) / n}$, the coefficient of the unsteady effect part of the governing boundary layer Eq. (12) which tends to be zero as $d \rightarrow \infty$. For the same reason, the coefficient of the terms $\left(1-F^{\prime 2}\right)$ will be zero. As a result, Eq. (12) is reduced to the Eq. (21) whose solution depends only on the values of $a$ and $n$, and it is, indeed, free from the unsteadiness parameter $\beta$. We note that the above result is possible only when $\beta$ is small in comparison to $d$. However, the high nonlinearity in Eq. (21) prohibits us to obtain the analytic solution of the same. But for $a=n=1$, the nonlinearity of this equation is removed and then it produces a closed form analytic solution (asymptotic suction profile) which is same as that of Schlichting and Gersten [24] (see equation (5.52) there).

Figure $7 \mathrm{a}$ and $\mathrm{b}$ show the asymptotic suction profiles for various values of $n$ when $a=1$. The novelty which arises from Fig. $7 \mathrm{a}$ is that the asymptotic suction profiles reach the free stream condition more gradually with the increasing degree of the shear-thinning nature of the fluid. As a result, the boundary layer thickness increases progressively as $n(<1)$ decreases which even extends to infinity when $n$ tends to zero. On the other hand, the boundary layer thickness decreases rapidly with an increasing value of $n(>1)$ and ultimately the suction profile gets terminated abruptly at a certain distance from the porous bounding surface depending upon the rheological behavior of the fluids. This result is clearly shown in Fig. 7b.

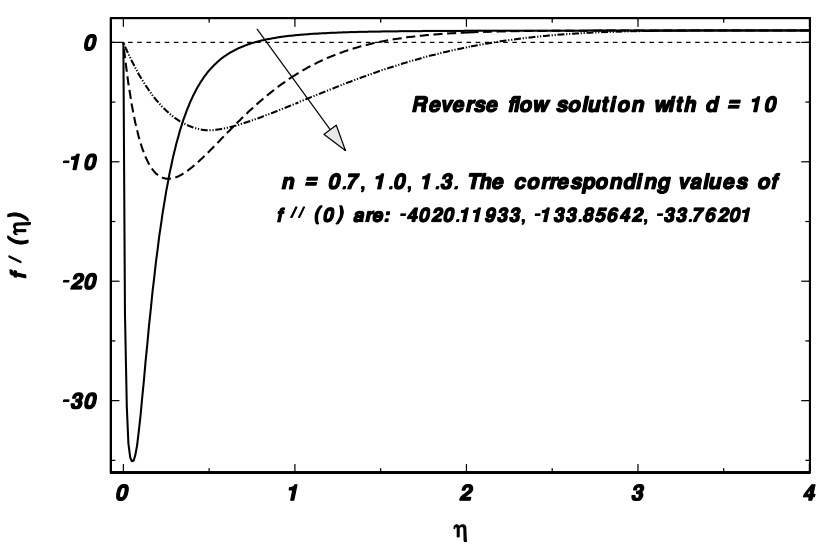

Fig. 8 Reverse flow solutions of (12) subjected to (13) for three fixed values of $n(=0.7,1.0$ and 1.3) in case of large value of suction $d(=10$, for example $)$
Fig. 7 Solutions of the asymptotic function $F(\omega)$ for strong suction (i.e., for $d \rightarrow \infty$ ) under various values of $n$ : (a) for $n<1$ and (b) for $n \geq 1$. Boundary layer thickness increases significantly with the decrease of $n<1$, while it decreases rapidly with an increasing value of $n>1$
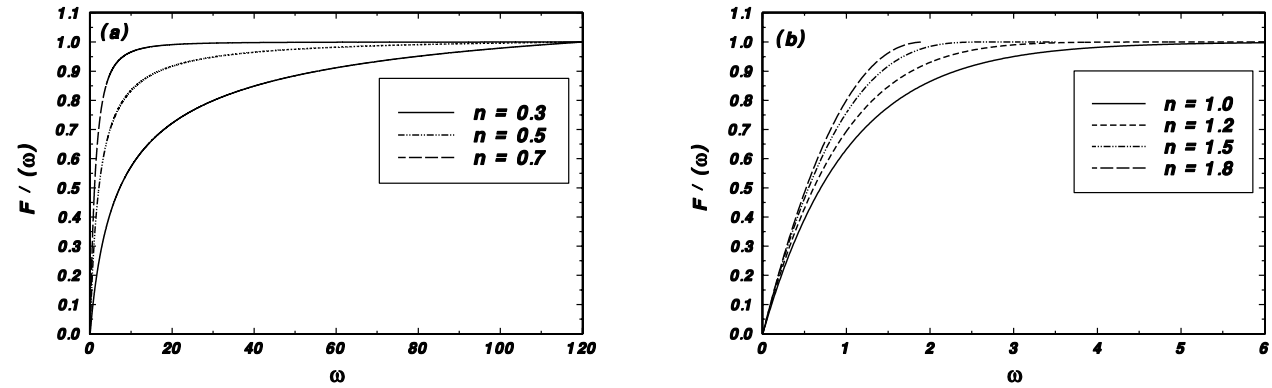

SN Applied Sciences a SPRINGer Nature journal 
Equation (21) is independent of $\beta$ which ensures the existence of a unique attached flow solution to this flow problem as the reverse flow solution exists only under a negative value of $\beta$ (Ref.14). Thus, we see that the Fig. 7 exhibits the attached flow solution for various values of $n$ as indicated on the figure. However, the reverse flow solution of this flow problem exists for large value of suction $d$, which have been mentioned earlier and shown in Fig. 8 for all types of fluids rheology. For pseudoplastic fluids $(n<1)$, the reverse flow solution jumps abruptly from the plate surface owing to the existence of very high negative value of the wall velocity gradient which is indicated on the figure. As a result, a very thin layer closer to plate surface as reflected in this figure always exists for this case.

\subsection{Effects of massive blowing $(d \ll-1)$}

For large blowing (i.e., for $d \rightarrow-\infty$ ) at the wall, another function $G(z)$ can be defined as follows:

$f(\eta)=d(1-G(z)) \quad$ where $\quad z=-\frac{\eta}{d}$.

With Eq. (23) and letting $d \rightarrow-\infty$, Eqs. (12) and (13) become of the forms:

$a\left(\frac{2 n}{n+1}\right)(G-1) \ddot{G}+a\left(1-\dot{G}^{2}\right)-\beta\left[\left(\frac{2-n}{1+n}\right) z \ddot{G}+\dot{G}-1\right]=0$,

$G(0)=0, \dot{G}(0)=0$ and $\dot{G}(z) \rightarrow 1$ as $z \rightarrow \infty$,

where a dot denotes the derivative with respect to the compressed variable $z$. Here, the term $\ddot{G}$ which describes the frictional forces, is discarded owing to the negligible influence of this term in this limiting situation. This reduces the order of the governing boundary layer equation which has now become a second order differential Eq. (24). The first two boundary conditions of (25) are the basic initial conditions for solving the Eq. (24), while the last condition of (25) is fulfilled at a finite value of $z$ (say, $z_{b}$ ) instead of $z \rightarrow \infty$ depending upon the rheological behavior of the fluids as well as the sum values of $(a+\beta)>0$. Therefore, in this case the last boundary condition of (25) will be of the form

$\dot{G}\left(z_{b}\right)=1$.

Table 1 gives the values of $z_{b}$ satisfying (26) for various values of $n$ when $\beta=-0.3$ and 0 . We have validated our present result with the corresponding result reported by Schlichting and Gersten [24] (shown inside the parenthesis for the row for $\beta=0$ ) for viscous fluid $(n=1)$, and have found a good agreement. From this table it is clear that the value of $z_{b}$ increases with $n$ when $\beta \leq 0$. We note that the above result is true for a given value of $\beta \leq 0$, while for $\beta>0$ the solution of (24) matches smoothly with the free boundary condition of (25) (see Fig. 10).

Using Eq. (24) along with the first two boundary conditions of (25), one can easily obtain the functional form of $\ddot{G}(0)$ as

$\ddot{G}(0)=\left(\frac{n+1}{2 n}\right)\left(\frac{a+\beta}{a}\right)$.

Equation (27) suggests that the sum value of $(a+\beta)$ will play the key role for the existence of the self-similar solutions to this flow problem since it can take any type of real value (positive, negative or zero). When $(a+\beta)=0$, Eq. (27) ensures that the value of $\ddot{G}(0)$ (i.e., $f^{\prime \prime}(0)$ ) is zero. This indicates that the boundary layer solutions to this flow problem will cease after a certain value of blowing depending upon the values of the viscosity index $n$. This result is exhibited in Figs. 9a and b which demonstrate the variation of $f^{\prime \prime}(0)$ against $d$ corresponding to three fixed values of $n(=0.5,1.0$ and 1.5). It is found that after a certain value of blowing both solutions (AFS and RFS) merge with one another to a value of zero velocity gradient. Below this critical value of blowing, there exists a unique AFS whose existence depends significantly on the values of $n$. Indeed when $(a+\beta)=0$, the pressure gradient which governs the flow inside the layer is zero (see Eq. (16) of Ref. 14), resulting in the demolition of the boundary layer solutions since they do not continue in a field of zero pressure gradient. We would now expect that after this critical value of blowing (for which both flows merge), the AFS flow, therefore, does not originate directly for the pressure gradient, rather for the dragging action of the velocity of the outer edge of the boundary layer. Indeed, this dragging action is more pronounced for shear-thinning fluids, and consequently one can get solution of this flow problem for very large rate of blowing with the decreasing value of $n<1$.

When $a=n=1$ and $\beta=-1$, Dholey and Gupta [12] investigated this case, and reported that after a fixed value of blowing $d(\approx-4.5)$, only the AFS continues and the solution becomes unique. But they did not present any supporting argument about the existence of this unique solution for large value of blowing. Here, we have made an attempt to mathematically establish that this unique attached flow solution does not continue for large value

Table 1 Values of $z_{b}$ for various values of $\beta$ and $n$ when $a=1$

\begin{tabular}{llllll}
\hline$\beta$ & $n \rightarrow 0.3$ & 0.5 & 1.0 & 1.2 & 1.5 \\
\hline-0.3 & 0.69752 & 0.98400 & 1.44189 & 1.56629 & 1.71657 \\
0.0 & 1.24998 & 1.39183 & $1.56985\left(\frac{\pi}{2}=1.57143\right)$ & 1.61356 & 1.66449 \\
\hline
\end{tabular}




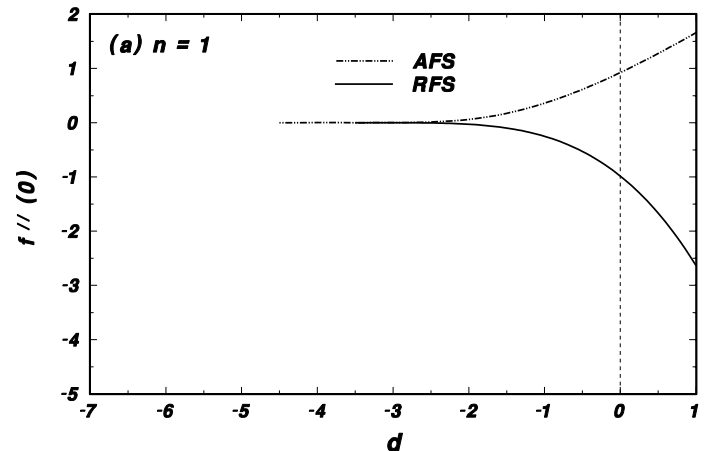

Fig. 9 Change of $f^{\prime \prime}(0)$ against $d$ for various values of $n$ which includes: (a) $n=1$ and (b) $n=0.5$ and 1.5 in cases of both AFS and RFS flows. The values of $f^{\prime \prime}(0)$ for both flows merge with one another to the value of zero for a definite value of blowing $d$ that

of blowing $d$. Most importantly, the zero value of $(a+\beta)$ along with (27) reduces the governing equation (24) to a homogeneous equation in $G(z)$ with homogeneous boundary conditions given as below:

$a\left(\frac{2 n}{n+1}\right)(G-1) \ddot{G}-a \dot{G}^{2}-\beta\left[\left(\frac{2-n}{1+n}\right) z \ddot{G}+\dot{G}\right]=0$,

$G(0)=0, \dot{G}(0)=0 \quad$ and $\quad \ddot{G}(0)=0$

Hence we can now confirm that the Eq. (28) subjected to (29) provides only the trivial solution $G(z)=0$ (i.e. $f(\eta)=d)$. Obviously, this solution does not satisfy the free boundary condition of (25). Hence we can conclude that for the fixed value of $(a+\beta)=0$ and after a definite value of blowing $d$, dependent on $n$, the governing boundary layer equation (12) yields only the inconsiderable

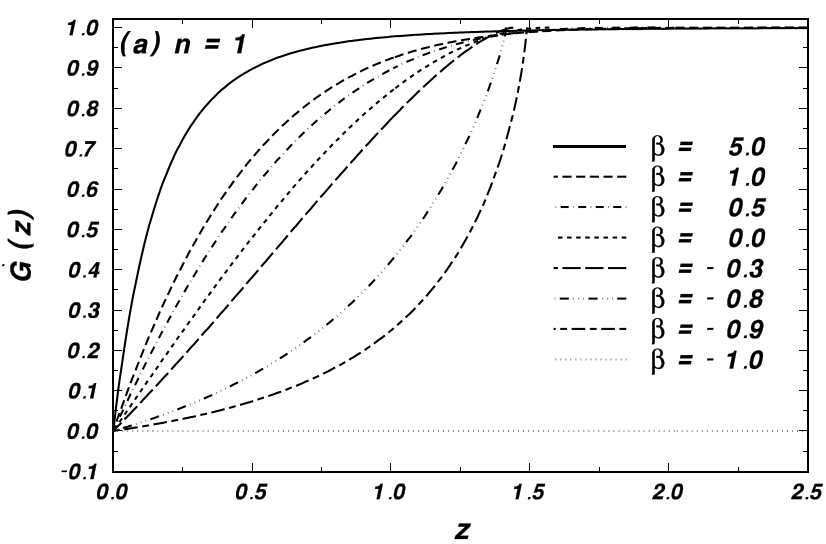

Fig. 10 Solutions of the asymptotic function $G(z)$ for massive blowing (i.e., for $d \rightarrow-\infty$ ) under various values of $\beta$ with two different values of $n$ : (a) $n=1$ and (b) $n=1.5$. The asymptotic profile approaches to the plate surface with an increasing value of $\beta>0$,

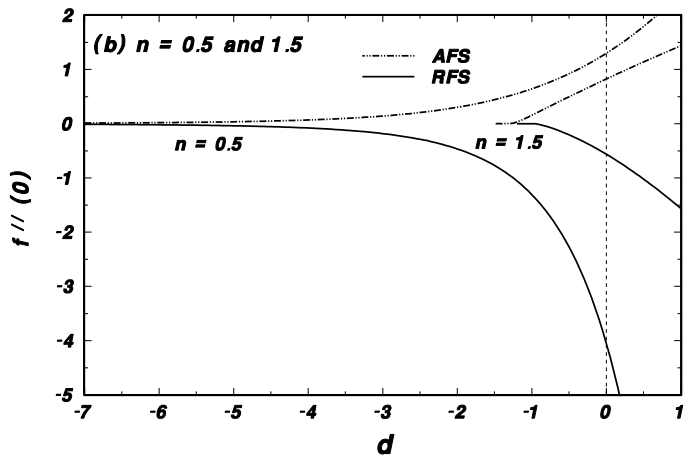

depends on the values of $n$. Below this value of blowing only AFS exists but up to a little more value of blowing $d$ owing to the dragging action of the outer potential flow

(worthless) solution $f(\eta)=d$ that does not gratify the free boundary condition of (13).

Solutions of Eq. (24) are shown in Fig. 10a and b for various values of $\beta$ when $a=1$ and $n=1.0$ and 1.5. The velocity profile $\dot{G}(z)$ continuously approaches the plate surface with the increase of $\beta>0$. But for $\beta<0$, the velocity profile moves further away from the plate surface and finally, it fulfills the outer boundary condition at a definite value of $z\left(=z_{b}\right.$, say) corresponding to a fixed value of $\beta(\approx-0.3)$. Below the value of $\beta \approx-0.3$, the velocity profile does not show the proper boundary layer flow characteristics and therefore it has no physical significance in the boundary layer theory. Interesting enough, the critical value of $\beta$ for both values of $n(=1.0$ and 1.5$)$ are nearly the same which is $\approx-0.3$ as reflected in these figures. This means that the critical value of $\beta$ relies weakly on the values of $n$. We note that the critical value of $\beta$ will decrease with an

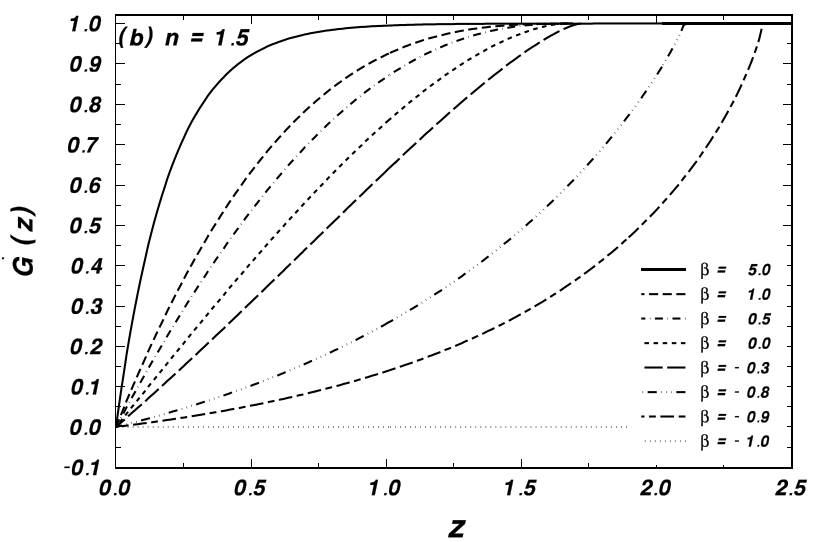

whereas it moves away from the surface with the decreasing value of $\beta<0$. The asymptotic profile for a negative value of $\beta$ (not too small) does not appear to have a boundary layer flow 
increasing value of $a$ since the flow accelerates with $a$. The uniqueness which emerges from these figures is that for $\beta=-1$, i.e., when $(a+\beta)=0$, Eq. (24) yields only the zero solutions $G(z)=0$, which we have mentioned earlier. The above results are also true for shear-thinning fluids $(n<1)$. Finally, we can conclude that for large rate of blowing the boundary layer solutions exist up to a definite value of $\beta(<0)$ which depends on the values of $a$ and $n$.

\section{Conclusions}

In this paper, an unsteady stagnation-point boundary layer flows of power-law fluids over a porous flat surface subjected to continuous suction or injection has been studied by numerically. The governing Navier-Stokes equations for a non-Newtonian fluid corresponding to the classical Ostwald-de Waele power-law model are converted into a third order non-linear ordinary differential equation by adapting the similarity transformations. This self-similar equation together with the relevant boundary conditions are then solved numerically by using the fourth-order Runge-Kutta integration technique for some values of the governing parameters. It is found that the influence of the mass transfer parameter $d$ in association with the viscosity index $n$ greatly changes the boundary layer flow dynamics. The analysis reveals that the shear-thinning fluids always yield a higher drag-coefficient than the shear-thickening fluids in comparison with the viscous fluid. As a consequence, the boundary layer separation is delayed with an increasing degree of the shear-thinning behavior of the fluids, whereas early separation is found in viscous as well as shear-thickening fluids. An important result of this analysis is the zero-displacement-thickness boundary layer profile which is possible for a suitable amount of suction $d$ that depends on the values of $n$. In this case, the boundary layer flow will be in the most stable condition since the frictional force does not affect the fluid motion by this amount of suction velocity. For a small amount of blowing $d$, significant amount of drag-coefficient is reduced which may be used in many engineering applications. Another remarkable result which emerges from this analysis is that the boundary layer solution of a dilatant fluid can match smoothly with the free boundary condition by a suitable amount of blowing $d$ depending upon the values of $a, \beta$ and $n$. For strong suction, the governing boundary layer equation is free from $\beta$, whereas it conceives the parameter $\beta$ for massive blowing imposed at the wall. However, both attached and reverse flow solutions exist for large values of suction $d$, whereas for large rate of blowing only the attached flow solution continues up to the certain critical values of blowing $d$ and $\beta(<0)$ which depend highly on the values of $a$ and $n$. Below this value of $\beta$, one can find the solution of the governing boundary layer equation of this flow problem which is physically non-admissible since it does not follow the proper boundary layer characteristics.

Acknowledgements The author is very thankful to the editors and referees for their constructive suggestions that essentially improved the presentation of the paper. The author would also like to convey thanks to Anita Dholey and Shreya Dholey for their kind cooperation during the work. This work has been supported by SERB (No. EMR/2016/005533) of India.

\section{Compliance with ethical standards}

Conflicts of interest The author declares that there is no conflict of interest.

\section{References}

1. Bird RB, Stewart WE, Lightfoot EN (1960) Transport phenomena. Wiley, New York

2. Schowalter WR (1960) The application of boundary layer theory to power-law pseudoplastic fluids : similar solutions. AIChE J 6:24-28

3. Acrivos A, Shah MJ, Petersen EE (1960) Momentum and heat transfer in laminar boundary layer flows of non-Newtonian fluids past external surfaces. AIChE J 6:312-317

4. Thompson ER, Snyder WT (1968) Drag reduction of a non-Newtonian fluid by fluid injection at the wall. J Hydron 2:177-180

5. Thompson ER, Snyder WT (1970) Laminar boundary layer flows of Newtonian fluids with non-Newtonian fluid injections. J Hydron 4:86-91

6. Teipel I (1974) Discontinuities in boundary layer problems of power-law fluids. Mech Res Commun 1:269-273

7. Pakdemirli M (1993) Boundary layer flow of power-law fluids past arbitrary profiles. IMA J Appl Math 50:133-148

8. Nachman A, Taliaferro S (1996) Mass transfer into boundary layers for power-law fluids. Proc R Soc Lond 365:313-326

9. Denier JP, Dabrowski PP (2004) On the boundary layer equations for power-law fluids. Proc R Soc Lond 460:3143-3158

10. Wel DM, Al-Ashhab S (2014) Similarity solutions for non-Newtonian power-law fluid flow. Appl Math Mech 35:1155-1166

11. Wu J, Thompson MC (1996) Non-Newtonian shear-thinning flows past a flat plate. J Non-Newton Fluid Mech 66:127-144

12. Dholey S, Gupta AS (2013) Unsteady separated stagnation-point flow of an incompressible viscous fluid on the surface of a moving porous plate. Phys Fluids 25(023601):1-16

13. Ma KHP, Hui WH (1990) Similarity solutions of the two-dimensional unsteady boundary layer equations. J Fluid Mech 66:537-559

14. Dholey S (2015) The boundary layers of an unsteady separated stagnation-point flow of a viscous incompressible fluid over of a moving plate. Fluid Dyn Res 47(035504):1-22

15. Lok YY, Pop I (2014) Stretching or shrinking for unsteady separated stagnation-point flow. Meccanica 49:1479-1492

16. Dholey S (2019) Unsteady separated stagnation-point flow over a permeable surface. Zeit Angew Math Physik 70:1-19

17. Xu H, Liao SJ (2005) Series solutions of unsteady magnetohydrodynamic flows of non-Newtonian fluids caused by an impulsively stretching plate. J Non-Newton Fluid Mech 159:46-55 
18. Cheng J, Liao SJ, Pop I (2005) Analytic series solutions of unsteady mixed convection boundary layer flow near the stagnation-point on a vertical surface in a porous media. J Porous Media 61:365-379

19. Xu H, Liao SJ, Pop I (2006) Series solutions of unsteady boundary layer flows of non-Newtonian fluids near a forward stagnation point. J Non-Newton Fluid Mech 139:31-43

20. Dholey $S$ (2018) On the fluid dynamics of unsteady separated stagnation-point flow of a power-law fluid on the surface of a moving flat plate. Euro J Mech B Fluids 70:102-114

21. Hiemenz K (1911) Die Grenzschicht an einem in den gleichförmigen Flüssigkeitsstorm eingetauchten geraden Kreiszylinder. Dingel Polytech J 326:321-410
22. Ralston A, Wilf HF (1968) Mathematical methods for digital computers. Wiley, New York

23. Rosenhead L (1963) Laminar boundary layer. Clarendon Press, Oxford

24. Schlichting H, Gersten $\mathrm{K}$ (2000) Boundary-layer theory. McGrawHill, New York

Publisher's Note Springer Nature remains neutral with regard to jurisdictional claims in published maps and institutional affiliations. 